\title{
Electron-electron interaction induced spin thermalization in quasi-low-dimensional spin valves
}

\author{
Tero T. Heikkilä \\ Low Temperature Laboratory, Aalto University School of Science and Technology, FI-00076 AALTO, Finland \\ Moosa Hatami, Gerrit E. W. Bauer \\ Kavli Institute of NanoScience, Delft University of Technology, 2628 CJ Delft, The Netherlands
}

Abstract

We study the spin thermalization, i.e., the inter-spin energy relaxation mediated by electron-electron scattering in small $\sim$ 'spin valves. When one or two of the dimensions of the spin valve spacer are smaller than the thermal coherence length, the direct spin energy exchange rate diverges and needs to be regularized by the sample dimensions. Here we consider two model systems: a long quasi-1D wire and a thin quasi-2D sheet.

Keywords: A. magnetic films and multilayers, D. thermoelectric effects, D. heat conduction, D. inter-spin heat 'exchange

PACS: 72.15.Jf,85.75.-d

\section{Introduction}

The thermoelectric response of a ferromagnet|normal metal|ferromagnet spin valve [1, 2, 3] depends sensitively on the strength of inter-spin energy relaxation (spin thermalization) inside the normal metal spacer 4]. In large structures at high temperatures, spin thermalization is dominated by electron-phonon coupling, whereas at low temperatures direct spin-flip scattering becomes important. A third mechanism is the electron-electron scattering, which in relatively large spin valves is weak and can typically be neglected. However, for smaller spin valves the electron-electron interaction becomes stronger. When one or more of the dimensions are smaller than the thermal coherence length $\xi_{T}=\sqrt{\hbar D /\left(k_{B} T\right)}$, the kernel of the electron-electron collision integral should be calculated for reduced $(1 \mathrm{D}$ or $2 \mathrm{D})$ spatial dimensions. In these cases the thermalization rate formally diverges [5, 6] and needs to be properly regularized. In this paper we discuss such regularization schemes and calculate the resulting thermalization rates.

\section{Theory}

In a biased spin valve with an antiparallel configuration of the magnetic reservoirs, the electron distribution function may depend on the spin index $\sigma$. In this case the electron-electron interactions can be described by the three different collision integrals represented by the diagrams in Fig. 1 and calculated as explained in the Appendix.

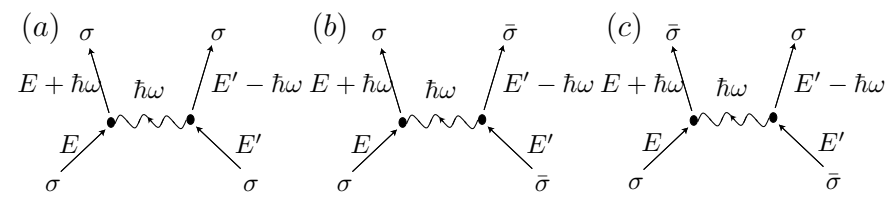

Figure 1: The three types of electron-electron scattering vertices in a system with spin-dependent distribution functions $f_{\sigma}(E)$. a) Equalspin scattering that does not lead to spin thermalization, b) spin conserving scattering and c) spin exchange scattering. The latter two cause spin thermalization and are discussed in this paper.

The kernels of the collision integrals depend on the Fermi liquid triplet parameter $F>-1$. The precise value of $F$ is important for the strength of spin thermalization caused by the electron-electron interactions: as shown below, close to the Stoner instability at $F=-1$ spin thermalization is quite strong, whereas it is much weaker for $F \approx 0$.

The inter-spin energy exchange can be described by calculating the spin thermalization heat current

$$
\dot{Q}=\nu_{F} \Omega \int d E E I^{\uparrow \downarrow}(E),
$$

where $I^{\uparrow \downarrow}$ is the collision integral for scattering between spin $\uparrow$ and spin $\downarrow$ electrons, $\nu_{F}$ is the density of states at the Fermi level and $\Omega$ is the volume of the spin valve spacer.

In the following we first discuss the resulting inter-spin thermalization due to the regular spin conserving term. This can be done for an arbitrary spin heat accumulation $T_{\uparrow}-T_{\downarrow}$, where $T_{\uparrow(\downarrow)}$ is the effective electron temperature 
for spin $\uparrow(\downarrow)$ electrons. Then we turn to the spin exchange term, concentrating on the linear response regime in which $T_{\uparrow}-T_{\downarrow} \ll\left(T_{\uparrow}+T_{\downarrow}\right) / 2$. Within the calculation, we limit ourselves to the case in which the difference between the chemical potentials of the two spin species, i.e., the spin accumulation in the spacer $\mu_{s}=\mu_{\uparrow}-\mu_{\downarrow} \approx 0$. This is because in the limit $\mu_{s} \ll T$ the effect of a finite $\mu_{s}$ on the inter-spin relaxation is quadratic in $\mu_{s}$.

For the electron-electron interaction, the effective dimensionality $(0 \mathrm{D}, 1 \mathrm{D}, 2 \mathrm{D}$ or $3 \mathrm{D})$ of the spin valve island depends on the ratio between the island thickness $d$, width $W$ and length $L$ to the thermal coherence length $\xi_{T}$ (without losing generality, we assume $d<W<L$ ). Alternatively, we can compare the temperature to the Thouless energies $\hbar D /(L, W, d)^{2}$ defined by these size scales. Therefore, 0d case is realized when $k_{B} T \ll \hbar D / L^{2}$, the $1 \mathrm{D}$ case when $\hbar D / L^{2} \ll k_{B} T \ll \hbar D / W^{2}$, the $2 \mathrm{D}$ case when $\hbar D / W^{2} \ll k_{B} T \ll \hbar D / d^{2}$ and the 3 D case when $k_{B} T \gg \hbar D / d^{2}$.

\section{Spin conserving term}

We assume that the spin-dependent electron distribution functions can be written in terms of the Fermi-Dirac functions with spin-dependent chemical potential $\mu_{\sigma}$ and temperature $T_{\sigma}$ [4], i.e., $f_{\sigma}(E)=f^{0}\left(E ; \mu_{\sigma}, T_{\sigma}\right)=\{1+$ $\left.\exp \left[\left(E-\mu_{\sigma}\right) /\left(k_{B} T_{\sigma}\right)\right]\right\}^{-1}$. In this case, we can use the standard relations 1

$$
\begin{aligned}
& f^{0}(E ; \mu, T)\left(1-f^{0}(E+x ; \mu, T)\right) \\
& =\left[f^{0}(E+x ; \mu, T)-f^{0}(E ; \mu, T)\right] n(-x ; T), \\
& \int d E\left[f^{0}(E ; \mu, T)-f^{0}(E+x ; \mu, T)\right]=x,
\end{aligned}
$$

where $n(x, T)=\left\{1-\exp \left[x /\left(k_{B} T\right)\right]\right\}^{-1}$ is the Bose distribution function. In the following, we use the short-hand notation $n_{\sigma}(x)=n\left(x ; T_{\sigma}\right)$. Using these relations, the spin conserving collision integral can be written in the form

$$
\begin{aligned}
I_{b}= & \frac{1}{2} \int d \omega \omega \mathcal{K}_{b}(\omega)\left[f_{\uparrow}(E)-f_{\uparrow}(E-\omega)\right] \\
& \times \underbrace{\left[\operatorname{coth}\left(\frac{\omega}{2 k_{B} T_{\downarrow}}\right)-\operatorname{coth}\left(\frac{\omega}{2 k_{B} T_{\uparrow}}\right)\right]}_{2\left[n_{\uparrow}(\omega) n_{\downarrow}(-\omega)-n_{\uparrow}(-\omega) n_{\downarrow}(\omega)\right]} .
\end{aligned}
$$

Here we used the symmetry of the kernel $\mathcal{K}_{b}(\omega)=\mathcal{K}_{b}(-\omega)$, by which the terms proportional to the chemical potentials $\mu_{\sigma}$ vanish. With a kernel $\mathcal{K}_{b}(\omega)=\kappa_{b}|\omega|^{\alpha}$, this yields for the spin conserving inter-spin heat current

$$
\dot{Q}_{b}=\frac{\nu_{F} \Omega \kappa_{b} \Gamma(4+\alpha) \operatorname{Li}_{4+\alpha}(1) k_{B}^{4+\alpha}}{\hbar^{\alpha+3}}\left(T_{\uparrow}^{4+\alpha}-T_{\downarrow}^{4+\alpha}\right)
$$

\footnotetext{
${ }^{1}$ We set $\hbar=k_{B}=1$ in the intermediate results, introducing them only in the final results.
}

where $\Gamma(x)$ is the gamma function and $\mathrm{Li}_{n}(x)$ is the polylogarithm function. Using the $\mathcal{K}_{b}(\omega)$ from Eqs. A.3 (A.5), we get

$$
\begin{aligned}
& \dot{Q}_{b}^{(1 \mathrm{D})}=\frac{3 \zeta\left(\frac{5}{2}\right) k_{B}^{5 / 2} L}{8 \sqrt{2 \pi} \hbar^{3 / 2} \sqrt{D(F+1)}}\left(T_{\uparrow}^{5 / 2}-T_{\downarrow}^{5 / 2}\right), \\
& \dot{Q}_{b}^{(2 \mathrm{D})}=\frac{\zeta(3) k_{B}^{3} A}{4 \pi D(F+1) \hbar^{2}}\left(T_{\uparrow}^{3}-T_{\downarrow}^{3}\right), \\
& \dot{Q}_{b}^{(3 \mathrm{D})}=\frac{15 \zeta\left(\frac{7}{2}\right) k_{B}^{7 / 2} \Omega}{32 \sqrt{2} \pi^{3 / 2} \hbar^{5 / 2}(D(F+1))^{3 / 2}}\left(T_{\uparrow}^{7 / 2}-T_{\downarrow}^{7 / 2}\right),
\end{aligned}
$$

where $\zeta(x)$ is the Riemann zeta-function and the superscript of $\dot{Q}$ indicates the dimensionality. This term is regular, but can in most cases be neglected since in $3 \mathrm{D}$ wires the spin thermalization due to electron-electron interactions is typically weaker than either the direct spin-flip scattering (at low temperatures) or electron-phonon scattering (at high temperatures) [4].

\section{Spin exchange term}

For the spin exchange term, the collision integral can be simplified to

$$
\begin{aligned}
& I_{c}=\frac{1}{2} \int d \omega d E^{\prime} \mathcal{K}_{c}(\omega)\left(f_{\uparrow}(E-\omega)-f_{\uparrow}\left(E^{\prime}-\omega\right)\right) \\
& \times\left(f_{\downarrow}\left(E^{\prime}\right)-f_{\downarrow}(E)\right) \underbrace{\left[\operatorname{coth}\left(\frac{E^{\prime}-E}{2 k_{B} T_{\downarrow}}\right)-\operatorname{coth}\left(\frac{E^{\prime}-E}{2 k_{B} T_{\uparrow}}\right)\right]}_{2\left[n_{\uparrow}\left(E^{\prime}-E\right) n_{\downarrow}\left(E-E^{\prime}\right)-n_{\uparrow}\left(E-E^{\prime}\right) n_{\downarrow}\left(E^{\prime}-E\right)\right]},
\end{aligned}
$$

leading to the inter-spin heat current

$$
\begin{aligned}
& \dot{Q}_{c}=\frac{\nu_{F} \Omega}{8} \times \\
& \int \frac{d E E d E^{\prime} d \omega \mathcal{K}_{c}(\omega) \sinh \left(\frac{\left(T_{\downarrow}-T_{\uparrow}\right)\left(E^{\prime}-E\right)}{2 T_{\downarrow} T_{\uparrow}}\right)}{\cosh \left(\frac{E+\omega}{2 T_{\downarrow}}\right) \cosh \left(\frac{E^{\prime}+\omega}{2 T_{\downarrow}}\right) \cosh \left(\frac{E-\mu_{s}}{2 T_{\uparrow}}\right) \cosh \left(\frac{E^{\prime}-\mu_{s}}{2 T_{\uparrow}}\right)} .
\end{aligned}
$$

This integral is more difficult to handle than Eq. (3). Therefore, we concentrate on the linear response limit $\dot{Q}_{c}=$ $K_{c}\left(T_{\uparrow}-T_{\downarrow}\right)$ and get

$$
K_{c}=\frac{\nu_{F} \Omega}{16} T^{3} \int \frac{d x d y d w \mathcal{K}_{c}(T w) x(x-y)}{\cosh \left(\frac{x}{2}\right) \cosh \left(\frac{y}{2}\right) \cosh \left(\frac{x+w}{2}\right) \cosh \left(\frac{y+w}{2}\right)} .
$$

The integral over $x$ and $y$ can be carried out analytically, leaving

$$
K_{c}=\frac{\nu_{F} \Omega T^{3}}{24} \int_{0}^{\infty} d w \frac{w^{2} \mathcal{K}_{c}(T w)\left(4 \pi^{2}+w^{2}\right)}{\sinh ^{2}\left(\frac{w}{2}\right)} .
$$

In the $3 \mathrm{D}$ case with $\mathcal{K}_{c} \propto|\omega|^{-1 / 2}$, this integral is regular and yields the result presented in Ref. [4]. However, in 
the $1 \mathrm{D}$ and $2 \mathrm{D}$ cases the integral over the first term has an infrared divergence and has to be regularized. In the following we present a simple regularization scheme based on the finite size of the sample.

Note that in Eq. (9) the cutoff scheme needs to be invoked only for the first term. We include also the second term in the $2 \mathrm{D}$ case, where the divergence is only logarithmic.

\subsection{D wire}

As discussed in the Appendix, in finite systems the kernels actually are sums over momenta. In the 1D wire the sum over the quantized momenta $q_{n}=2 \pi n / L$, where $L$ is the length of the wire, can be carried out directly. In this case the characteristic scale for the frequency is given by the Thouless energy $E_{T}^{1 \mathrm{D}}=D / L^{2}$. When $|\omega| \gg E_{T}^{1 \mathrm{D}}$, we recover Eq. A.3. For $|\omega| \ll E_{T}^{1 \mathrm{D}}$, the kernel does not depend on $\omega$,

$$
\mathcal{K}_{c}\left(|\omega| \ll E_{T}^{1 \mathrm{D}}\right)=\frac{\pi^{2} F^{2}}{180 \Omega\left(E_{T}^{1 \mathrm{D}}\right)^{2}(1+F)^{2}} .
$$

We can hence use $E_{T}^{1 \mathrm{D}}$ as a lower cutoff in Eq. (9). In principle, we should also include the part of the kernel which is constant for low frequencies $\omega \lesssim E_{T}^{1 \mathrm{D}}$. However, the contribution to the integral from this part is of the order of $\left(E_{T}^{1 \mathrm{D}} / T\right)^{3 / 2}$ times the contribution of the second part, and can hence be disregarded in the $1 \mathrm{D}$ limit $E_{T}^{1 \mathrm{D}} \ll$ $T$. The remaining integral is $K_{c} \propto I_{1}$ with

$$
I_{1}=\int_{E_{T}^{1 \mathrm{D}} / T}^{\infty} \frac{\sqrt{w} d w}{\sinh ^{2}(w / 2)}
$$

In the limit $k_{B} T \gg E_{T}^{1 \mathrm{D}}$ the most significant contribution comes from frequencies $w \ll 1$ for which $\sinh (w) \approx w$, leading to

$$
I_{1} \approx 8 \sqrt{\frac{T}{E_{T}^{1 \mathrm{D}}}}
$$

Including this contribution, we obtain for the spin thermalization heat conductance from the spin exchange contribution

$$
K_{c}^{1 \mathrm{D}}=\frac{F^{2} k_{B} 4 \sqrt{2} \pi\left(k_{B} T\right)^{2}}{3 \hbar E_{T}^{1 \mathrm{D}}(F+2)(F+\sqrt{F+1}+1)},
$$

where we reintroduced $k_{B}$ and $\hbar$. The diverging term hence gives rise to a temperature dependent scaling as $\propto T^{2}$.

As discussed in Ref. [4], a spin valve can be characterized in terms of the temperature above which spin thermalization is stronger than heat diffusion through the contacts, i.e., $K_{c} \gg \mathcal{L}_{0} G_{0} T$, where $G_{0}$ is the spin-averaged contact conductance and $\mathcal{L}_{0}=\pi^{2} k_{B}^{2} /\left(3 e^{2}\right)$ is the Lorenz number. This characteristic temperature for electron-electron interaction in a $1 \mathrm{D}$ sample is

$$
T_{\mathrm{ch}, \mathrm{e}-\mathrm{e}}^{1 \mathrm{D}}=\frac{(F+2)(F+\sqrt{F+1}+1)}{16 \sqrt{2} F^{2}} \frac{E_{T}^{1 \mathrm{D}}}{k_{B}} g,
$$

where $g=G_{0} /\left(e^{2} / h\right)$ is the dimensionless conductance of the contacts. Let us estimate the characteristic temperature and the thermal coherence length $\xi_{T}$. The latter is

$$
\xi_{T} \approx 85 \mathrm{~nm} \times\left(\frac{D}{0.001 \mathrm{~m}^{2} / \mathrm{s}} \frac{1 \mathrm{~K}}{T}\right)^{1 / 2} .
$$

Wires with lateral dimensions less than $\xi_{T}$ fall into the $1 \mathrm{D}$ limit. Connecting such wires to reservoirs via contacts with resistance $10 \Omega$ and assuming $F=-0.3$, we get

$$
T_{\mathrm{ch}, \mathrm{e}-\mathrm{e}}^{1 \mathrm{D}} \stackrel{F=0.3}{\approx} 25 \mathrm{~K} \times\left(\frac{D}{0.001 \mathrm{~m}^{2} / \mathrm{s}}\left(\frac{1 \mu \mathrm{m}}{L}\right)^{2} \frac{G_{0}}{0.1 \mathrm{~S}}\right) .
$$

For wires fabricated from materials close to the Stoner instability, such as $\mathrm{Pd}$, say with $F=-0.9$, this characteristic temperature is

$$
T_{\mathrm{ch}, \mathrm{e}-\mathrm{e}}^{1 \mathrm{D}} \stackrel{F=-0.9}{\approx} 0.5 \mathrm{~K} \times\left(\frac{D}{0.001 \mathrm{~m}^{2} / \mathrm{s}}\left(\frac{1 \mu \mathrm{m}}{L}\right)^{2} \frac{G_{0}}{0.1 \mathrm{~S}}\right) .
$$

These values should be compared to the characteristic temperature due to the electron-phonon interaction using the results from Ref. [4]. This is

$T_{\mathrm{ch}, \mathrm{e}-\mathrm{ph}} \approx 4 \mathrm{~K} \times\left(\frac{10^{9} \mathrm{Wm}^{-3} \mathrm{~K}^{-5}}{\Sigma} \frac{0.015(\mu \mathrm{m})^{3}}{\Omega} \frac{G_{0}}{0.1 \mathrm{~S}}\right)^{-1 / 3}$.

In this case electron-electron interaction in systems close to the Stoner instability is the dominating spin thermalization mechanism.

Another way to characterize the spin energy exchange is via the spin thermalization time $\tau_{\mathrm{st}}=\mathcal{L}_{0} e^{2} \nu_{F} T \Omega /\left(2 K_{\mathrm{e}-\mathrm{e}}\right)$. From Eq. (13):

$$
\tau_{\mathrm{st}, \mathrm{e}-\mathrm{e}}^{1 \mathrm{D}}=\frac{\pi(F+2)(F+\sqrt{F+1}+1) \hbar \nu_{F} \Omega}{8 \sqrt{2} F^{2}} \frac{E_{T}^{1 \mathrm{D}}}{k_{B} T} .
$$

This scattering time should be compared to the direct spinflip time of roughly $100 \mathrm{ps}$ in typical samples [7]. With $F=-0.3$ and some other typical values for metals with wire cross section $A=W d, \tau_{\text {st,e-e }}$ is rather long,

$$
\begin{aligned}
\tau_{\mathrm{st}, \mathrm{e}-\mathrm{e}}^{1 \mathrm{D}} & \stackrel{F}{ }={ }^{\approx}-0.31 \mathrm{~ns} \\
& \times\left[\frac{\nu_{F}}{10^{47} \mathrm{~J}^{-1} \mathrm{~m}^{-3}} \frac{A}{1500 \mathrm{~nm}^{2}} \frac{1 \mu \mathrm{m}}{L} \frac{D}{0.001 \mathrm{~m}^{2} / \mathrm{s}} \frac{1 \mathrm{~K}}{T}\right]
\end{aligned}
$$

whereas for $F=-0.9$ it is

$$
\begin{aligned}
\tau_{\mathrm{st}, \mathrm{e}-\mathrm{e}}^{1 \mathrm{D}} & \stackrel{F=-0.9}{\approx} 20 \mathrm{ps} \\
& \times\left[\frac{\nu_{F}}{10^{47} \mathrm{~J}^{-1} \mathrm{~m}^{-3}} \frac{A}{1500 \mathrm{~nm}^{2}} \frac{1 \mu \mathrm{m}}{L} \frac{D}{0.001 \mathrm{~m}^{2} / \mathrm{s}} \frac{1 \mathrm{~K}}{T}\right] .
\end{aligned}
$$

These examples show that the electron-electron interaction in $1 \mathrm{D}$ samples is especially relevant for systems close to the Stoner instability. 


\section{2. $2 D$ square sheet}

In the two-dimensional case $\mathcal{K}_{c}=\kappa_{2 D}|\omega|^{-1}$ and the resulting integral has a logarithmic divergence. Now the regular term in Eq. (9) yields

$$
\nu_{F} \Omega \zeta(3) \kappa_{2 D} T^{2}
$$

and for the diverging term, we need to evaluate the integral

$$
\begin{aligned}
I_{2} & =\int_{E_{T} / T}^{\infty} \frac{w d w}{\sinh ^{2}(w / 2)} \\
& =2 \frac{E_{T}}{T} \operatorname{coth}\left(\frac{E_{T}}{2 T}\right)-2 \ln \left[2 \cosh \left(\frac{E_{T}}{T}\right)-1\right] \\
& \approx 4\left[\ln \left(\frac{T}{E_{T}}\right)+1\right]
\end{aligned}
$$

Here the lower cutoff $E_{T}=\hbar D / W^{2}$ is the confinement energy due to the smaller of the two extended dimensions. Hence, for the full spin thermalization heat conductance we get

$$
K_{c} \stackrel{2 \mathrm{D}}{=} \frac{F^{2} k_{B}^{3} T^{2}\left(4 \pi^{2}\left(\ln \left(\frac{T}{E_{T}}\right)+1\right)+6 \zeta(3)\right)}{12 \pi \hbar E_{T}^{2 \mathrm{D}}\left(F^{2}+3 F+2\right)},
$$

where $E_{T}^{2 \mathrm{D}}=\hbar D /(L W)$.

In this case the characteristic temperature is

$T_{\mathrm{ch}, \mathrm{e}-\mathrm{e}}^{2 \mathrm{D}}=\frac{(F+1)(F+2) g}{4 F^{2} W\left(\frac{(F+1)(F+2) g e^{\frac{3(F(2 F+3)+6) \zeta(3)}{4 \pi^{2} F^{2}}+1}}{2 F^{2}} \frac{E_{T}^{2 \mathrm{D}}}{E_{T}}\right)} \frac{E_{T}^{2 \mathrm{D}}}{k_{B}}$,

where $W(x)$ is the principal solution for $z$ in $x=z e^{z}$. Here we included also the regular terms from Eqs. (5b) and (20) besides the term proportional to $\ln \left(T / E_{T}\right)$. To estimate this characteristic temperature, we consider a square bar of area (width $\times$ length) $A=1(\mu \mathrm{m})^{2}$ and much thinner than $\xi_{T}$. In this case we have $T_{\mathrm{ch}, \mathrm{e}-\mathrm{e}}^{2 \mathrm{D}} \approx 10 \mathrm{~K}$ for wires with $F=-0.3$ and $T_{\mathrm{ch}, \mathrm{e}-\mathrm{e}}^{2 \mathrm{D}} \approx 0.3 \mathrm{~K}$ with $F=-0.9$ and otherwise similar values as in the above $1 \mathrm{D}$ case. For comparison, with the thickness $d=30 \mathrm{~nm}$, we get for the phonon contribution $T_{\mathrm{ch}, \mathrm{e}-\mathrm{ph}} \approx 3.2 \mathrm{~K}$.

The spin thermalization time is

$$
\begin{aligned}
& \tau_{\mathrm{st}, \mathrm{e}-\mathrm{e}}^{2 \mathrm{D}}= \\
& \frac{4 \pi^{3}(F+1)(F+2) \nu_{F} \Omega \hbar}{\left(8 \pi^{2} F^{2}\left(\log \left(\frac{k_{B} T}{E_{T}}\right)+1\right)+3\left(2+F+4 F^{2}\right) \zeta(3)\right)} \frac{E_{T}^{2 \mathrm{D}}}{k_{B} T} .
\end{aligned}
$$

Assuming a film thickness of $30 \mathrm{~nm}$, for $F=-0.3$ we get $\tau_{\mathrm{st}, \mathrm{e}-\mathrm{e}} \approx 7$ ns whereas for $F=-0.9 \tau_{\mathrm{st}, \mathrm{e}-\mathrm{e}} \approx 80 \mathrm{ps}$ at $T=1 \mathrm{~K}$ and otherwise similar conditions as above.

\section{Conclusions}

One of the key features enabling the success of spintronics in the past two decades is the long spin-flip time

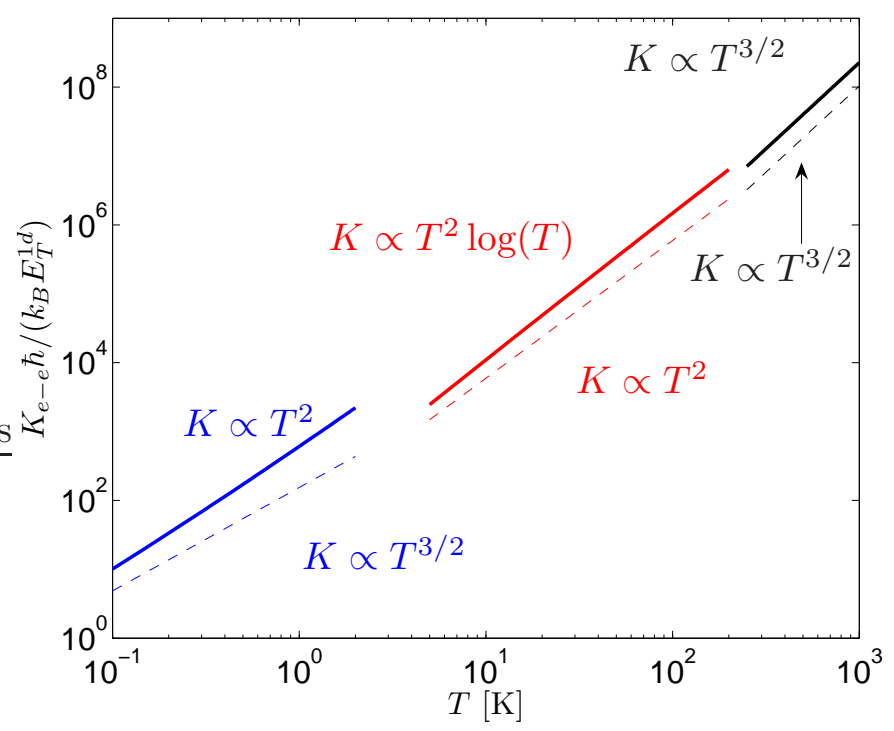

Figure 2: (Color online): Temperature dependence of the spin thermalization heat conductance due to electron-electron interaction for a wire with length $1 \mu \mathrm{m}$, width $100 \mathrm{~nm}$ and thickness $10 \mathrm{~nm}$, diffusion constant $D=0.03 \mathrm{~m}^{2} / \mathrm{s}$ and Fermi liquid parameter $F=-0.3$ (solid lines) and $F=0$ (dashed lines). The three regimes plotted in the figure are for the $1 \mathrm{D}, 2 \mathrm{D}$ and $3 \mathrm{D}$ limits. The heat conductance is written in units of $k_{B} E_{T}^{1 \mathrm{D}} / \hbar$, where $E_{T}^{1 \mathrm{D}} / k_{B}=22 \mathrm{mK}$, corresponding to the crossover temperature between $0 \mathrm{~d}$ and $1 \mathrm{D}$ behavior. In the case $F=0$ the spin exchange contribution vanishes, which changes the temperature dependences of the heat conductances in the $1 \mathrm{D}$ and $2 \mathrm{D}$ cases.

found in metals. One recent trend has been the study of thermal transport in spintronic systems. Special interest has been devoted to the study of magnetothermal effects, where the heat transport depends on the magnetic configuration of the system. Besides the long spin relaxation time, such effects rely on relatively weak interspin energy relaxation. This was studied in detail by the present authors in Ref. [4]. It was found, among other things, that making the sample smaller reduces the effects of inter-spin relaxation. However, the conventional 3D theory of electron-electron interaction in disordered metals applies only when the system dimensions exceed the thermal coherence length $\xi_{T}$. Moreover, it was pointed out in Refs. [5, 6] that the electron-electron contribution to the energy relaxation in reduced dimensions diverges. In this paper we have addressed this divergence by introducing a natural cutoff emerging from the sample size. The main results of this consideration, Eqs. (13) and (20), show that indeed the electron-electron interactions in $1 \mathrm{D}$ and $2 \mathrm{D}$ samples are more relevant for the spin thermalization than in the $3 \mathrm{D}$ case. However, for typical normal metal spacers for which the Fermi liquid triplet parameter $F$ is far from the Stoner instability $F=-1$, this effect is still masked by direct spin-flip processes at low temperatures and electron-phonon relaxation at high temperatures. The situation is different for materials close to the Stoner instability, for example palladium wires doped with nickel. In these systems the inter-spin energy relaxation due to 
electron-electron interaction may be the dominating relaxation mechanism.

The behavior of the spin thermalization heat conductance due to electron-electron scattering is illustrated in Fig. 2. It shows the regimes of different dimensionality for an example system.

The theory presented in this manuscript is based on the collision integrals calculated in Ref. [5], which generalize the Altshuler-Aronov theory [8] to the spin-dependent case. We point out that the electron-electron energy relaxation described by this theory in the 1D limit has been investigated experimentally in detail [9]. However, according to these experiments the measured energy relaxation is stronger by roughly an order of magnitude than that predicted by the theory. This is evidence that the results for the spin thermalization conductances presented in Eqs. (5a), (5b), (13) and (20) might be underestimated.

\section{Acknowledgments}

This work was supported by the Academy of Finland, the Finnish Cultural Foundation, and NanoNed, a nanotechnology programme of the Dutch Ministry of Economic Affairs. TTH acknowledges the hospitality of Delft University of Technology, where this work was initiated.

\section{Appendix A. Collision integrals and kernels}

The collision integrals describing electron-electron scattering in spin-dependent systems are

$$
\begin{aligned}
& I_{a}(E)=\int d \omega d E^{\prime} \mathcal{K}_{a}(\omega) \times \\
& {\left[\left(1-f_{\sigma}(E)\right)\left(1-f_{\sigma}\left(E^{\prime}\right)\right) f_{\sigma}(E-\omega) f_{\sigma}\left(E^{\prime}+\omega\right)\right.} \\
& \left.-f_{\sigma}(E) f_{\sigma}\left(E^{\prime}\right)\left(1-f_{\sigma}(E-\omega)\right)\left(1-f_{\sigma}\left(E^{\prime}+\omega\right)\right)\right] \\
& I_{b}(E)=\int d \omega d E^{\prime} \mathcal{K}_{b}(\omega) \times \\
& {\left[\left(1-f_{\sigma}(E)\right)\left(1-f_{-\sigma}\left(E^{\prime}\right)\right) f_{\sigma}(E-\omega) f_{-\sigma}\left(E^{\prime}+\omega\right)\right.} \\
& \left.-f_{\sigma}(E) f_{-\sigma}\left(E^{\prime}\right)\left(1-f_{\sigma}(E-\omega)\right)\left(1-f_{-\sigma}\left(E^{\prime}+\omega\right)\right)\right] \\
& I_{c}(E)=\int d \omega d E^{\prime} \mathcal{K}_{c}(\omega) \times \\
& {\left[\left(1-f_{\sigma}(E)\right)\left(1-f_{-\sigma}\left(E^{\prime}\right)\right) f_{\sigma}\left(E^{\prime}-\omega\right) f_{-\sigma}(E+\omega)\right.} \\
& \left.-f_{\sigma}(E) f_{-\sigma}\left(E^{\prime}\right)\left(1-f_{\sigma}\left(E^{\prime}-\omega\right)\right)\left(1-f_{-\sigma}(E+\omega)\right)\right] .
\end{aligned}
$$

The kernels $K_{a, b, c}(\omega)$ depend on the dimensionality of the sample [5]:

$$
\begin{aligned}
\mathcal{K}_{a} & =\frac{8}{2 \pi \nu_{F} \Omega} \sum_{\mathbf{q}} \frac{1}{\omega^{2}+\left(D \mathbf{q}^{2}\right)^{2}} \frac{\left(\frac{1}{2}+F\right)^{2}+\frac{\omega}{\left(2 D \mathbf{q}^{2}\right)^{2}}}{(1+F)^{2}+\frac{\omega^{2}}{\left(D \mathbf{q}^{2}\right)^{2}}} \\
\mathcal{K}_{b} & =\frac{8}{2 \pi \nu_{F} \Omega} \sum_{\mathbf{q}} \frac{1}{\omega^{2}+\left(D \mathbf{q}^{2}\right)^{2}} \frac{\frac{1}{4}+\frac{\omega}{\left(2 D \mathbf{q}^{2}\right)^{2}}}{(1+F)^{2}+\frac{\omega^{2}}{\left(D \mathbf{q}^{2}\right)^{2}}} \\
\mathcal{K}_{c} & =\frac{8}{2 \pi \nu_{F} \Omega} \sum_{\mathbf{q}} \frac{1}{\omega^{2}+\left(D \mathbf{q}^{2}\right)^{2}} \frac{F^{2}}{(1+F)^{2}+\frac{\left(\omega-F \mu_{s}\right)^{2}}{\left(D \mathbf{q}^{2}\right)^{2}}}
\end{aligned}
$$

Here $D$ is the diffusion constant in the spacer and $F$ the Fermi liquid interaction parameter in the triplet channel.

In this paper we describe systems with at least one extended dimension, i.e., quasi-1D, quasi-2D or 3D metal islands. According to the standard prescriptions for calculating the sums

$$
\begin{gathered}
\sum_{\mathbf{q}} \mapsto L /(\pi) \int_{0}^{\infty} d q, \quad d=1 \\
\sum_{\mathbf{q}} \mapsto A /(2 \pi) \int_{0}^{\infty} q d q, \quad d=2 \\
\sum_{\mathbf{q}} \mapsto \Omega /\left(2 \pi^{2}\right) \int_{0}^{\infty} q^{2} d q, \quad d=3,
\end{gathered}
$$

we get for $d=1$

$$
\begin{aligned}
\mathcal{K}_{a} & =\frac{\frac{4 F(F+1)}{(F+2)(\sqrt{F+1}+1)}+1}{2 \pi A \nu_{F} \sqrt{2 D(F+1)}} \frac{1}{|\omega|^{3 / 2}} \\
\mathcal{K}_{b} & =\frac{1}{2 \pi A \nu_{F} \sqrt{2 D(F+1)}} \frac{1}{|\omega|^{3 / 2}} \\
\mathcal{K}_{c} & =\frac{\sqrt{2} F^{2}}{\pi A \nu_{F} \sqrt{D(F+1)} \mathcal{C}} \\
& \stackrel{\mu_{s} \rightarrow 0}{\rightarrow} \frac{\sqrt{2} F^{2}}{\pi A(F+2)(\sqrt{F+1}+1) \nu_{F}|\omega|^{3 / 2} \sqrt{D(F+1)}}
\end{aligned}
$$

Here $\mathcal{C}=\left(\sqrt{\left|\omega-F \mu_{s}\right|}+\sqrt{F+1} \sqrt{|\omega|}\right)\left(\left|\omega-F \mu_{s}\right|+(F+\right.$ 1) $|\omega|)$. This result was also obtained in Ref. [5]. For $d=2$ the kernels are

$$
\begin{aligned}
\mathcal{K}_{a} & =\frac{1+2 F^{2}+\frac{5 F}{2}}{\pi D\left(4 F^{2}+12 F+8\right) d \nu_{F}|\omega|} \\
\mathcal{K}_{b} & =\frac{1}{8 \pi D(F+1) d \nu_{F}|\omega|} \\
\mathcal{K}_{c} & =\frac{F^{2}}{2 \pi D(F+1) d \nu_{F}\left(\left|\omega-F \mu_{s}\right|+(F+1)|\omega|\right)} \\
& \stackrel{\mu_{s} \rightarrow 0}{\rightarrow} \frac{F^{2}}{2 \pi D\left(F^{2}+3 F+2\right) d \nu_{F}|\omega|}
\end{aligned}
$$


and for $d=3$ we get

$$
\begin{aligned}
\mathcal{K}_{a} & =\frac{1+\frac{4(F+1)(\sqrt{F+1} F+\sqrt{F+1}-1)}{F+2}}{2 \pi^{2} \nu_{F} \sqrt{|\omega|}(2 D(F+1))^{3 / 2}} \\
\mathcal{K}_{b} & =\frac{1}{2 \pi^{2} \nu_{F} \sqrt{|\omega|}(2 D(F+1))^{3 / 2}} \\
\mathcal{K}_{c} & =\frac{F\left(|(F+1) \omega|^{3 / 2}-\left|\omega-F \mu_{s}\right|^{3 / 2}\right)}{\sqrt{2} \pi^{2} \nu_{F}(D(F+1))^{3 / 2}\left(\mu_{s}+\omega\right)\left((F+2) \omega-F \mu_{s}\right)} \\
& \stackrel{\mu_{s} \rightarrow 0}{\rightarrow} \frac{F\left((F+1)^{2}-\sqrt{F+1}\right)}{\sqrt{2} \pi^{2} D^{3 / 2}(F+1)^{2}(F+2) \nu_{F} \sqrt{|\omega|}} .
\end{aligned}
$$

In the present paper, we only use the kernels with $\mu_{s} \rightarrow 0$. This is because in the limit $\mu_{s} \ll T$ the effect of a finite $\mu_{s}$ is quadratic in $\mu_{s}$.

\section{References}

\section{References}

[1] M. Hatami, G. E. W. Bauer, Q. Zhang, P. J. Kelly, Phys. Rev. Lett. 99(6) (2007) 066603.

[2] M. Hatami, G. E. W. Bauer, Q. Zhang, P. J. Kelly, Phys. Rev. B 79 (2009) 174426.

[3] Y. Dubi, M. D. Ventra, Phys. Rev. B 79 (2009) 081302.

[4] T. T. Heikkilä, M. Hatami, G. E. W. Bauer, Phys. Rev. B 81, (2010) 100408(R).

[5] O. Dimitrova, V. Kravtsov, JETP Lett. 86 (2007) 670.

[6] N. M. Chtchelkatchev, I. S. Burmistrov, Phys. Rev. Lett. 100 (2008) 206804.

[7] F. J. Jedema, H. B. Heersche, A. T. Filip, J. J. A. Baselmans, B. J. van Wees, Nature 416 (2002) 713.

[8] B. Altshuler, A. Aronov, in: A. Efros, M. Pollak (Eds.), ElectronElectron Interactions in Disordered Systems, Elsevier, Amsterdam, 1985.

[9] B. Huard, A. Anthore, F. Pierre, H. Pothier, N. O. Birge, D. Esteve", Solid State Commun. 131 (2004) 599 - 607. 